\title{
ULTRABRIGHT single-photon sources
}

\author{
Pascale SENELLART ${ }^{1}$ \\ Valérian GIESZ \\ Loic LANCO ${ }^{1,2}$ \\ ${ }^{1}$ Centre de Nanosciences \\ et de Nanotechnologies (C2N), \\ CNRS, Route de Nozay, \\ 91460 Marcoussis, France \\ ${ }^{2}$ Université Paris 7 Diderot, \\ 75205 Paris Cedex 13, France \\ pascale.senellart@lpn.cnrs.fr
}

\section{Inefficient quantum light sources}

One major hurdle in the development of quantum optical technologies is the manufacture of efficient single-photon sources. This type of source should ideally emit a single photon on demand, in a well-defined mode of the electromagnetic field ${ }^{1}$. At present, optical quantum communications and computation protocols use heralded single-photon sources, where a pair of photons is generated by nonlinear parametric frequency conversion, and the detection of one
The development of a quantum communication network will require sources that efficiently emit single photons. Now, using a new lithography technique that garnered a silver CNRS medal in 2014 , it has recently proved possible to fabricate these sources using quantum dots (QDs), that is, artificial solid-state atoms. Performed at cryogenic temperatures, this technique makes it possible to position a single QD in the middle of an optical microcavity with nanometric precision.

photon signals the arrival of the other. To avoid generating too many light pulses containing more than one photon, these sources operate at flows where less than $5 \%$ of pulses contain a pair of photons, the others being essentially empty. This situation is far from ideal, as it dramatically reduces the rate of optical communication and, in the broader field of quantum computation, restricts the complexity of the calculations that can be performed.

\section{Semiconductor quantum dots offer new promise..}

The past 15 years have seen the emergence of another approach to fabricating these sources. It involves the use of nano-emitters, known as semiconductor QDs. One semiconductor material of nanometric size is inserted in another, forming a highly effective

An attenuated laser is not a source of single photons. Although the photons are emitted in a defined field mode, the photon statistics are Poissonian. This means that even when the pulses contain less than one photon on average, the probability of a pulse containing two is non-negligible.

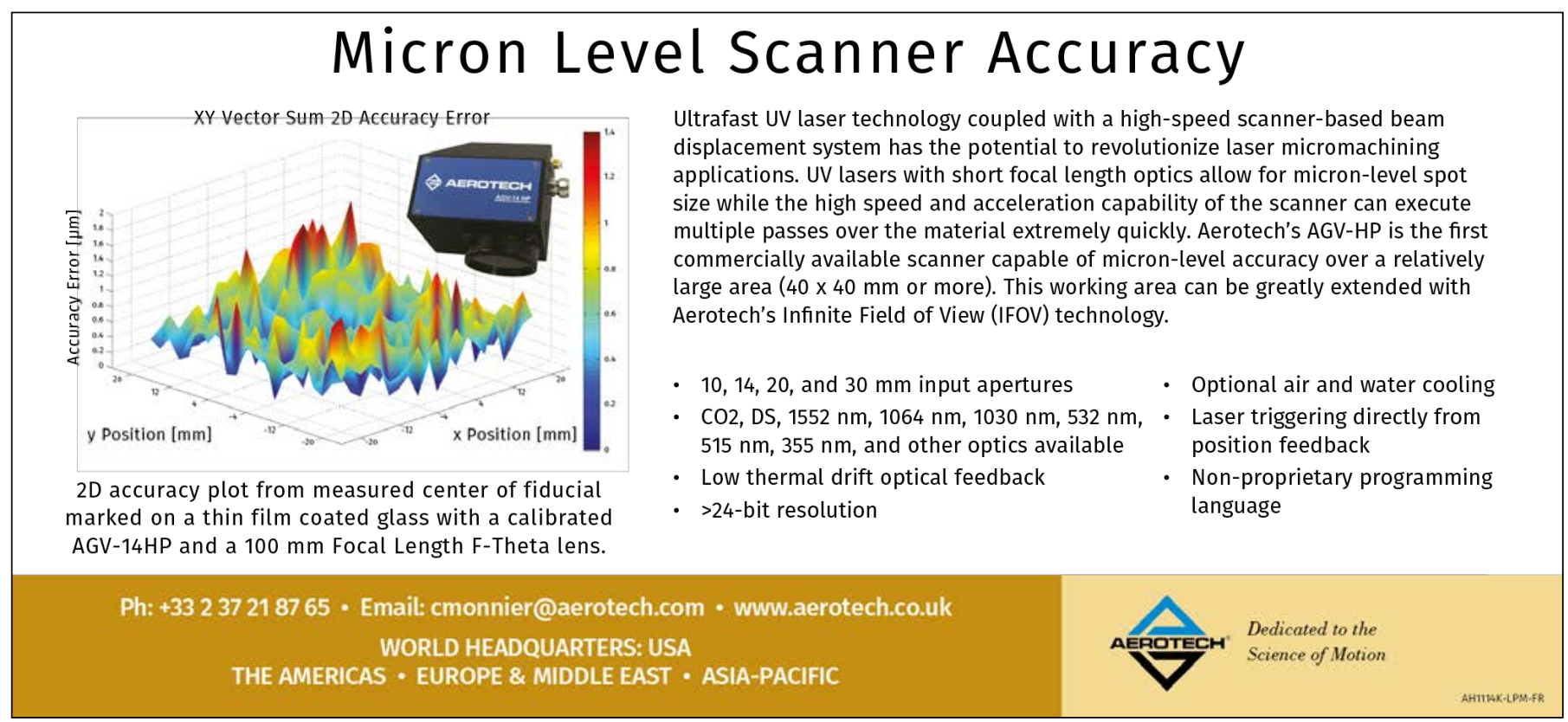



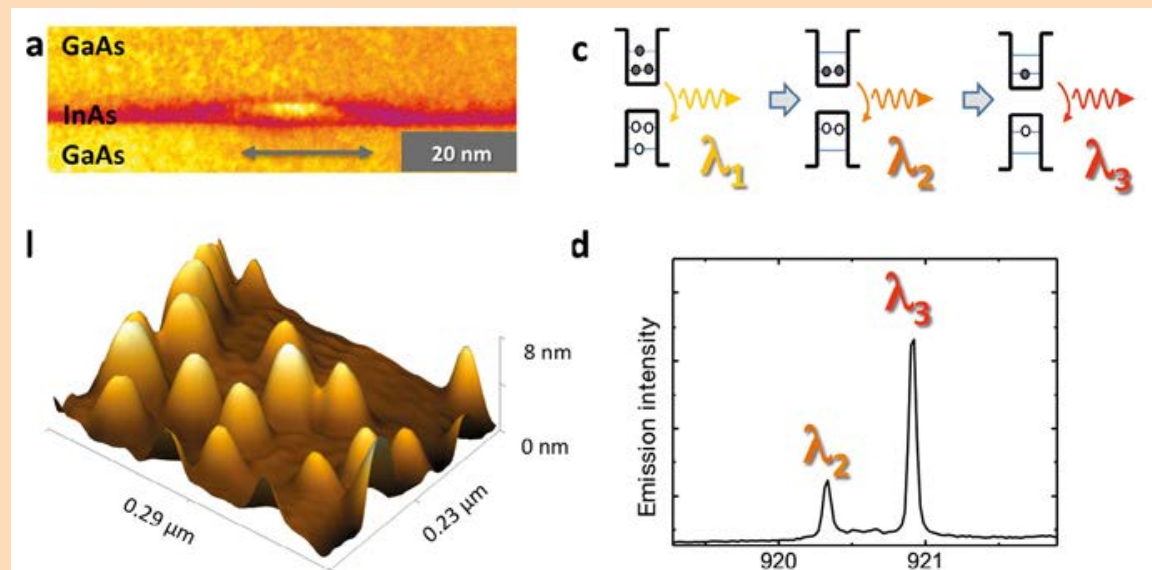

d

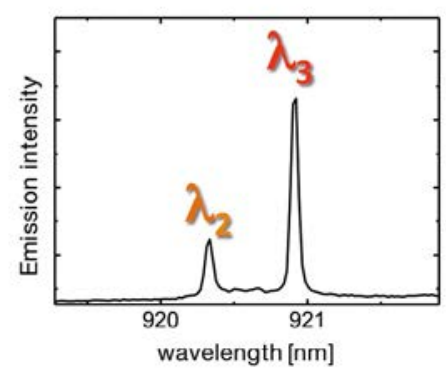

Figure 1. Semiconductor quantum dots. (a) Transmission electron microscope image of a single quantum dot (with the kind permission of G. Patriarche and A. Lemaître, C2NCNRS). (b) Atomic-force microscope image of a plane of quantum dots (with the kind permission of C. Gomez-Carbonell, C2N-CNRS). (c) Functional diagram of single-photon emission: when several electron-hole pairs are created in the quantum dot, the system emits several photons one after the other at different wavelengths. (d) Typical emission spectrum of a quantum dot at $10 \mathrm{~K}$.

electron trap [1]. The carriers in this type of nanostructure have discrete energy levels, like the electronic states in a single atom. When a large number of carriers are created in a $\mathrm{QD}$, the system goes back to its ground state by emitting successively several photons. Their emission wavelength depends directly on the number of carriers in the $\mathrm{QD}$, so that at a given wavelength, on one photon is emitted. These objects are ideal single-photon sources, in that for each excitation pulse, there is an almost $100 \%$ probability that the QD will emit a single photon [2].

The problem is that they suffer from the same drawback as all semiconductor light sources, namely the difficulty of collecting the emitted light, which is mostly trapped inside the material by total internal reflection. Although the $\mathrm{QD}$ does indeed emit a photon at each excitation, less than $1 \%$ actually emerge from the device. This problem can be overcome by controlling the way the QD is coupled to light. As E. Purcell predicted in 1946, spontaneous emission is not an intrinsic property of the emitter, but depends on its electromagnetic environment [3]. Placing a point emitter in an optical cavity can increase its coupling to a particular mode of the optical field and accelerate its spontaneous emission in this mode. For this to happen, two conditions must be met: the QD must be placed at the confined optical field maximum, and must be in spectral resonance with it.

\section{... but how can we control them?}

Meeting these conditions has proved challenging. QDs self-assemble from differences in lattice parameters when indium arsenide is grown in a gallium arsenide matrix. These QDs are therefore totally randomly located. To make matters worse, they measure just 20-30 nm wide and are only a few $\mathrm{nm}$ thick, corresponding to ten or so atomic layers. The slightest variation in their shape or composition therefore significantly changes their emission wavelength. In this context, traditional fabrication methods, which cannot control the QDs' spatial and spectral positions, have yields below $0.1 \%$.

A great deal of time and effort has been devoted to solving this problem. Some researchers have tried to force QDs to grow on predefined networks. However, these promising methods have yet to result in QDs with satisfactory optical qualities. Other techniques that have been tried involve using atomic force microscopy to detect individual QDs. Each one is mapped with respect to gold markers, and electron-beam lithography is used to fabricate a cavity aligned with these markers [4]. Further technological stages are then required to fine-tune the nanocavity mode into resonance with the quantum dot exciton. These techniques are quite spectacular, although they are difficult to accomplish and have not been reproduced.

\section{Is in situ photolithography the solution?}

The Center for Nanoscience and Nanotechnology (C2N) of the French National Research Centre (CNRS) has come up with an original approach whereby the location of a QD is optically measured, and a cavity centred on this QD is defined using laser lithography [5].

To achieve this, a photoresist is deposited on a sample containing self-assembled QDs. This sample is placed in a cryostat to lower its temperature to $10 \mathrm{~K}$, so that the QDs emit extremely pure single photons. A red laser beam excites QD emission without exposing the photoresist (Fig. 2a-(I)). The diameter of the laser's excitation spot is limited by diffraction (approx. one micron), but the excitation of the nanometric-sized QD depends on local excitation density. This means that by moving the emitter within the excitation beam to maximize its emission, it is possible to measure the QD's position (Fig. 2a-(II)). Just as the summit of a mountain several kilometres wide can be located to within one metre, a QD can be localized to within $50 \mathrm{~nm}$. The next stage in this in situ lithography technique involves using a green laser, accurately superimposed on the red laser, to expose a pattern in the photoresist centred on the QD, in order to directly define the optical cavity (Fig. 2a-(III)). Finally, the cavity's geometry is adjusted so that it is precisely tuned with the QD's known emission wavelength. These localization and writing stages can be repeated, enabling a large number of 
devices to be manufactured in a single lithography stage. The cavities are then etched using techniques normally employed to etch optoelectronic devices.

First demonstrated with micropillar cavities, produced by etching a cylinder measuring a few microns in diameter from a planar Bragg cavity sample, in situ lithography has since been used to accurately place QDs in various types of cavities. To enhance the technique's potential, the CNRS has teamed up with the private company attocube to develop an in situ lithography technology that has been used since 2010 to make more complex structures, including so-called connected pillar cavities allowing electrical control of the device [6], coupled cavities [7], and hybrid plasmonic-dielectric cavities.

\section{Ultrabright single- photon sourices}

Inserting a $Q D$ in a cavity makes it possible to modify its spontaneous emission and fabricate extremely bright single-photon sources. This may look like an oxymoron, but as the QD emits a photon at each optical excitation, brightness refers here to the probability of collecting that photon. In the absence of a cavity, this brightness is hardly few percent.

When it is coupled with a cavity, the QD's emission rate in the cavity mode is accelerated by a factor $F_{P}$, whereas in other optical modes this emission rate remains unchanged. The probability of the QD emitting its single photon in the cavity mode is thus $p=F_{P} /\left(F_{P}+1\right)$. With a moderate $\left(F_{P}=3-5\right)$ acceleration of spontaneous emission, the probability is nearly $75-85 \%$. With a judicious choice of cavity geometry, it is now possible to produce in a controlled and reproducible fashion sources that emit single photons on demand with $80 \%$ brightness [8]. These sources are 20 times brighter than any of those used today in the field of optical quantum information. Moreover, as it takes a QD approximately one hundred picoseconds to emit

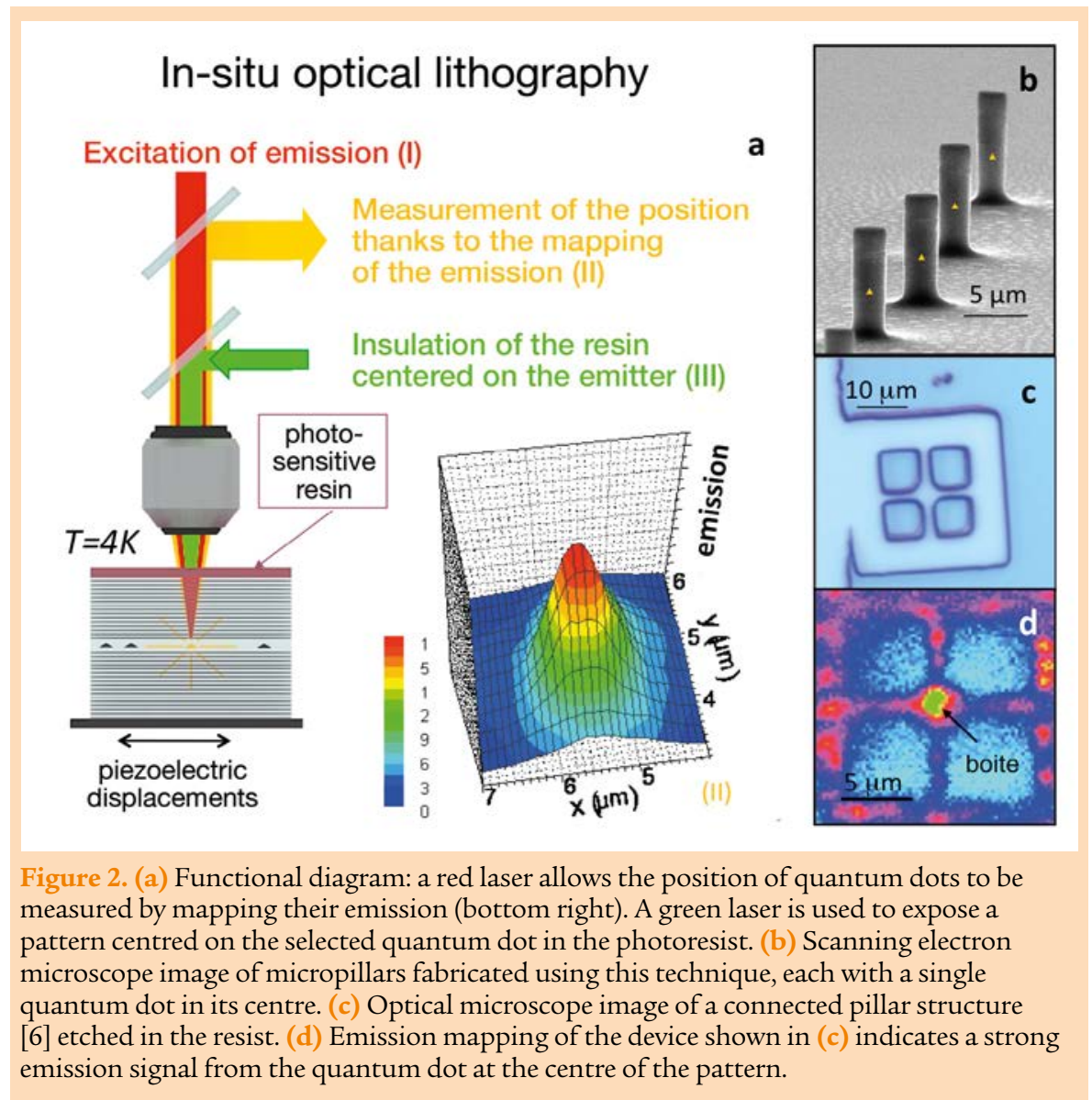

\section{CMS series Multispectral cameras}

Small footprint $\left(52 \times 52 \times 22 \mathrm{~mm}^{3}\right)$

Low weight $(<60 \mathrm{~g})$

8 spectral bands $+1 \mathrm{~B} \& \mathrm{~W}$

20 to $40 \mathrm{~nm}$ spectral resolution $1.3 \mathrm{Mpx}$ raw resolution USB 3.0 - 10bits
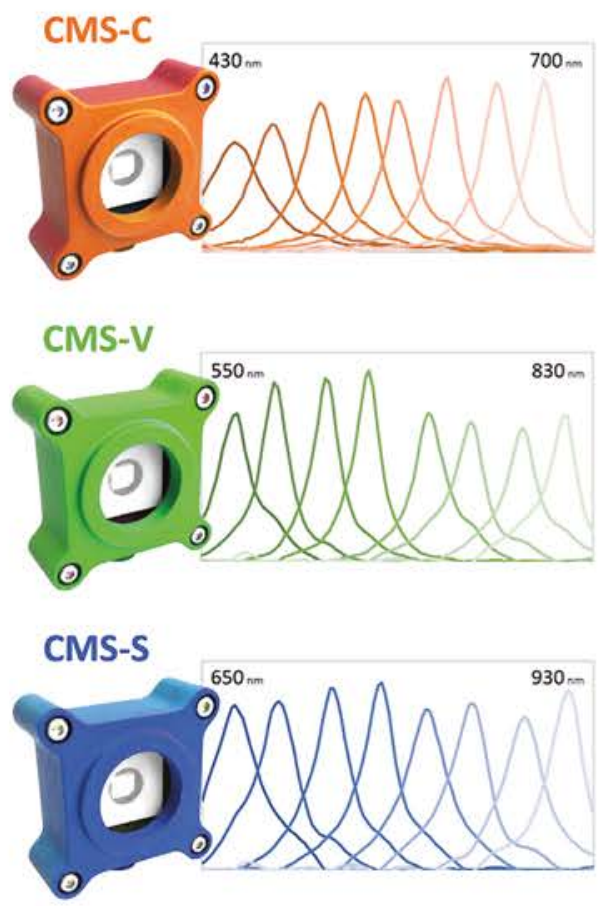

\section{Get from images both spectral and spatial information for your applications :}

$\checkmark$ Bio-medical
$\checkmark$ Surgery
$\checkmark$ Precision Farming
$\checkmark$ Environment
$\checkmark$ Cosmetics
$\checkmark$ Dermatology
$\checkmark$ Autonomous vehicles
$\checkmark$ Surveillance
$\checkmark$ Security
$\checkmark$...

\section{For more information}

Web : www.silios.com

Email : contact-cms@silios.fr 
a single photon, these sources can be used at frequencies up to the $\mathrm{GHz}$ range. Most recently, they have been shown to generate highly indistinguishable photons, a key requirement for many quantum applications [9].

\section{A whole new potential to explore}

These single-photon sources could potentially bring about a critical increase in the complexity of quantum computation, as well as in the speed of quantum communications. Andrew White's group in Brisbane, Australia, a founding group in optical quantum technologies, embarked on this adventure 3 years ago. Working closely with this group, the $\mathrm{C} 2 \mathrm{~N}$ team has already set up a quantum logic gate with an ultrabright QD source [10]. More recently, they have demonstrated first Boson sampling measurements using the $\mathrm{C} 2 \mathrm{NQD}$ sources, demonstrating a 200 speed-up in the manipulation of three photons as compared to the same measurement conducted with usual sources [11].

In situ lithography does not serve solely to fabricate efficient single-photon sources. With clever cavity geometry, it has proved possible to make the brightest source of polarization-entangled photon pairs so far [7]. Just as we can efficiently collect the single photons emitted by a QD, so we can ensure that every photon sent to the device interacts with the QD. This property has allowed us to demonstrate optical nonlinearities on the scale of just a few photons, thus opening the way up to the implementation of logic gates on the single-photon scale [12]. Moreover, inserting a charge carrier in the QD creates a device that can rotate the polarization of a photon by several degrees, depending on whether this carrier is in a up- or a down-spin state [13]. From this point on, many things are possible. Fabricated with optoelectronic tools, these devices could, for example, allow the development of solid-state quantum networks where the photons generated by QDs act as flying qubits, linking spin quantum memories! -
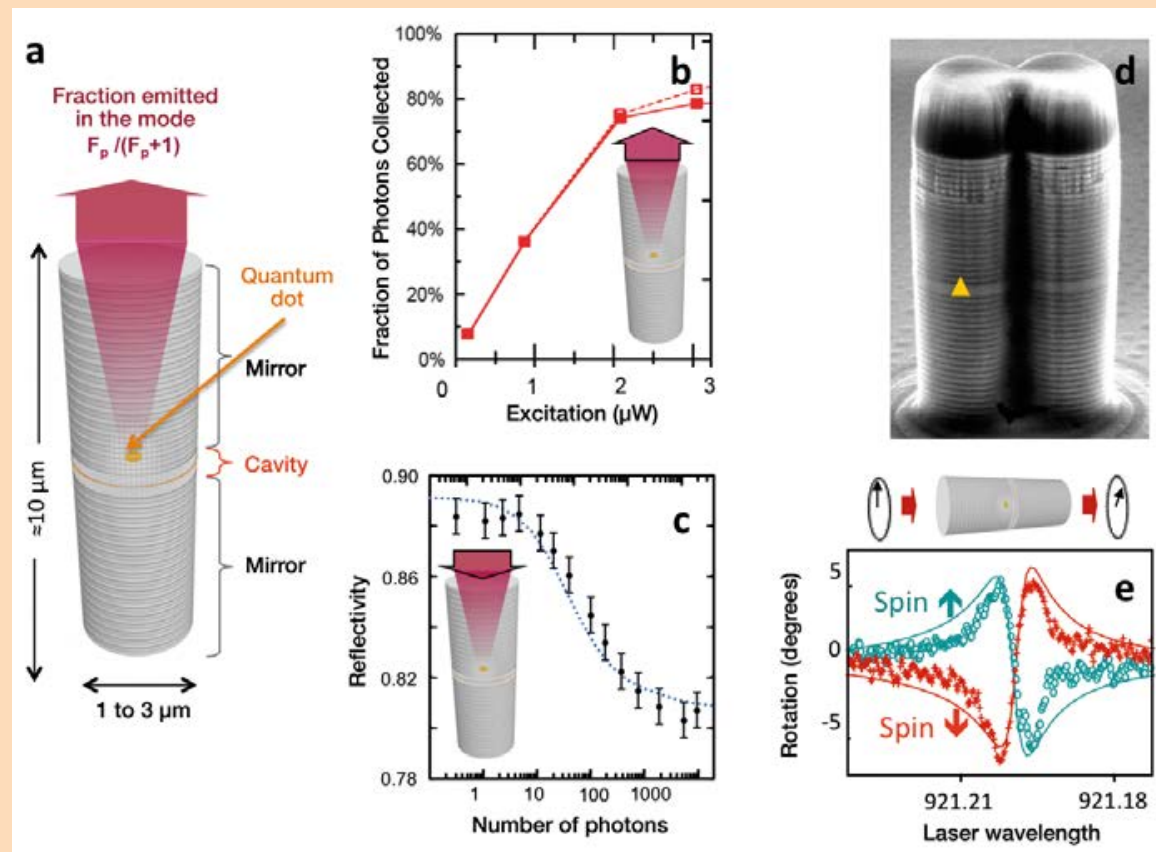

Figure 3. (a) Functional diagram of a micropillar cavity and light extraction through acceleration of spontaneous emission. (b) Probability of collecting the single photon emitted by the quantum dot according to excitation power. At saturation, brightness is $80 \%$ [8]. (c) Demonstration of an optical nonlinearity obtained for just 8 photons by measuring quantum-dot cavity system reflectivity [12]. (d) Electron microscope image of an ultrabright source of polarization-entangled electron pairs [7]. (e) Photon polarization rotation of $\pm 6^{\circ}$ induced by a single spin in the cavity, depending on whether the spin is up or down [13].

\section{FURTHER READING}

[1] J.-Y. Marzin, J.-M. Gérard, A. Izraël, D. Barrier, G. Bastard, Phys. Rev. Lett. 73, 716 (1994)

[2] P. Michler, A. Kiraz, C. Becher, W.V. Schoenfeld, P.M. Petroff, Lidong Zhang, E. Hu, A. Imamoglu, Science 290, 2282 (2000)

[3] E.M. Purcell, Phys. Rev. 69, 674 (1946)

[4] A. Badolato, K. Hennessy, M. Atatüre, J. Dreiser, P.M. Petroff, A. Imamoglu, Science 308, 1158 (2005)

[5] A. Dousse, L. Lanco, J. Suffczynski, E. Semenova, A. Miard, A. Lemaître, I. Sagnes, C. Roblin, J. Bloch, P. Senellart, Phys. Rev. Lett. 101, 267404 (2008)

[6] A.K. Nowak, S.L. Portalupi, V. Giesz, O. Gazzano, C. Dal Savio, P.-F. Braun, K. Karrai, C. Arnold, L. Lanco, I. Sagnes, A. Lemaître, P. Senellart, Nature Commun. 5, 3240 (2014)

[7] A. Dousse, J. Suffczynski, A. Beveratos, O. Krebs, A. Lemaître, I. Sagnes, J. Bloch, P. Voisin, P. Senellart, Nature 466, 217 (2010)

[8] O. Gazzano, S. Michaelis de Vasconcellos, C. Arnold, A.K. Nowak, E. Galopin, I. Sagnes, L. Lanco, A. Lemaitre, P. Senellart, Nature Commun. 4, 1425 (2013)

[9] N. Somaschi, V. Giesz, L. De Santis, J.C. Loredo, M. P. Almeida, G. Hornecker, S. L. Portalupi, T. Grange, C. Antón, J. Demory, C. Gómez, I. Sagnes, N.D. Lanzillotti-Kimura, A. Lemaítre, A. Auffeves, A.G. White, L. Lanco \& P. Senellart, Nature Photonics 10, 340-345 (2016)

[10] O. Gazzano, M.P. Almeida, A.K. Nowak, S.L. Portalupi, A. Lemaitre, I. Sagnes, A.G. White, P. Senellart, Phys. Rev. Lett. 110, 250501 (2013)

[11] J.C. Loredo, M.A. Broome, P. Hilaire, O. Gazzano, I. Sagnes, A. Lemaitre, M.P. Almeida, P. Senellart, A.G. White, arXiv:1603.00054

[12] V. Loo, C. Arnold, O. Gazzano, A. Lemaître, I. Sagnes, O. Krebs, P. Voisin, P. Senellart, L. Lanco, Phys. Rev. Lett. 109, 166806 (2012)

[13] C. Arnold, J. Demory, V. Loo, A. Lemaitre, I. Sagnes, M. Glazov, O. Krebs, P. Voisin, P. Senellart, L. Lanco, Nature Commun. 6, 6236 (2015) 